Psychotherapeut 2018 63:145-152 https://doi.org/10.1007/s00278-017-0245-1 Online publiziert: 19. Oktober 2017 (c) Der/die Autor(en) 2017. Dieser Artikel ist eine Open-Access-Publikation.

\section{Redaktion}

M. Cierpka, Heidelberg

B. Strauß, Jena

CrossMark

\author{
Michaela Hiebler-Ragger ${ }^{1,2}$. Christina Gollner ${ }^{3}$ Petra Klampfl $f^{3}$ \\ Liselotte Nausner ${ }^{3} \cdot$ Human-Friedrich Unterrainer ${ }^{1,2,3,4}$ \\ ' Universitätsklinik für Psychiatrie und Psychotherapeutische Medizin, Medizinische Universität Graz, Graz, \\ Österreich \\ ${ }^{2}$ Zentrum für Integrative Suchtforschung - Verein Grüner Kreis, Wien, Österreich \\ ${ }^{3}$ Fachsektion Integrative Gestalttherapie, Österreichischer Arbeitskreis für Gruppentherapie und \\ Gruppendynamik, Wien, Österreich \\ ${ }^{4}$ Institut für Psychologie, Karl-Franzens-Universität Graz, Graz, Österreich
}

\title{
Integrative Gestalttherapie in Österreich
}

\section{Ausbildungs- und Berufsbedingungen, Kohärenzgefühl und Burn-out}

\begin{abstract}
Evaluation und Qualitätssicherung in der Psychotherapie nehmen wachsenden Raum ein. Wichtig ist hierzu die differenzierte Betrachtung der Ausbildungslandschaft und der Arbeitsbedingungen von PsychotherapeutInnen, die wesentlich zu deren psychischem Wohlbefinden und beruflicher Entwicklung beitragen. Der retrospektiven Einschätzung von Input-, Prozess- und Output-Variablen der Psychotherapieausbildung kommt dabei große Bedeutung zu, da so die nachhaltige Relevanz von Ausbildungsbestandteilen erfasst werden kann und AbsolventInnen als LehrtherapeutInnen und/oder im Rahmen der Berufspolitik Einfluss auf die zukünftige Gestaltung der Ausbildung nehmen können.
\end{abstract}

\section{Hintergrund}

Nach wie vor gibt es wenige Studien, die sowohl verschiedene Aspekte der Psychotherapieausbildung als auch die Persönlichkeit und die berufliche Weiterentwicklung von PsychotherapeutInnen untersuchen. Auch wenn in den vergangenen Jahrzehnten oft auf die Notwendigkeit der Ausbildungsforschung aufmerksam gemacht wurde, gestaltet sich die Literatur in diesem Bereich noch recht unübersichtlich und heterogen. Während beispielsweise die große innereuropäische Diskrepanz in den Rahmenbedingungen der Psychotherapieausbildung bekannt ist und folglich die Notwendigkeit wissenschaftlich fundierter, übergreifender Ausbildungsstandards angeführt wird (Strauß und Kohl 2009a), gibt es bisher kaum veröffentlichte Studien zu den spezifischen Ausbildungsbedingungen und der allgemeinen professionellen Entwicklung von PsychotherapeutInnen in Österreich (Hagleitner und Lang 2005).

\section{Psychotherapieausbildung}

Grundsätzlich kann in der Psychotherapieausbildung zwischen Input, Prozess und Output unterschieden werden (Strauß und Kohl 2009b). Als Input wird die Ausgangslage der TeilnehmerInnen (Motive für den Ausbildungswunsch, Kosten etc.) verstanden. Hierzu können auch die Vorkenntnisse zur Therapierichtung (in Bezug auf Menschenbild, Persönlichkeitstheorie etc.) gezählt werden. Der Prozess umfasst die Entwicklung und die Umsetzung von Fertigkeiten. Hierbei spielen, wie beispielsweise von Laireiter und Botermans (2005) näher erläutert, folgende Aspekte der Ausbildung eine wesentliche Rolle: (a) theoretische Inhalte und Methoden, (b) eigene psychotherapeutische Praxis, (c) Supervision und (d) Lehrtherapie bzw. Selbsterfahrung. Der Output schließlich umfasst unmittelbare und langfristige Ergebnisse des Ausbildungsprozesses, wie professionelle Identität und Weiterentwicklung (Strauß und Kohl 2009b). Ronnestad und Skovholt (2013) führen dazu an, dass ein wissenschaftlich fundiertes Model über den gesamten Verlauf der professionellen Entwicklung noch ebenso fehlt wie die Definition einer notwendigen Wissensbasis für PsychotherapeutInnen.

\section{Arbeitszufriedenheit: Belastungen und Ressourcen}

Im Laufe ihrer professionellen Entwicklung sind PsychotherapeutInnen andauernden Belastungen ausgesetzt. Diese liegen beispielsweise im emotionalen Aufwand der Begegnungen mit KlientInnen sowie in den hohen Anforderungen an Haltung und Persönlichkeit der PsychotherapeutInnen (Hoffmann und Hoffmann 2008; Lups et al. 2012). Nach Jaeggi (2001) erleben viele PsychotherapeutInnen ihre Berufswahl als Berufung, woraus sich hohe Ansprüche an die eigene Leistungsfähigkeit ergeben. Kanfer et al. (2012) sehen in diesem übersteigerten Engagement und in der hohen emotionalen Beteiligung die Ursache eines möglichen Burn-outs, das wiederum die Qualität von Psychotherapien beeinträchtigen kann. Demnach meint Burn-out „... den Verlust positiver Emp- 


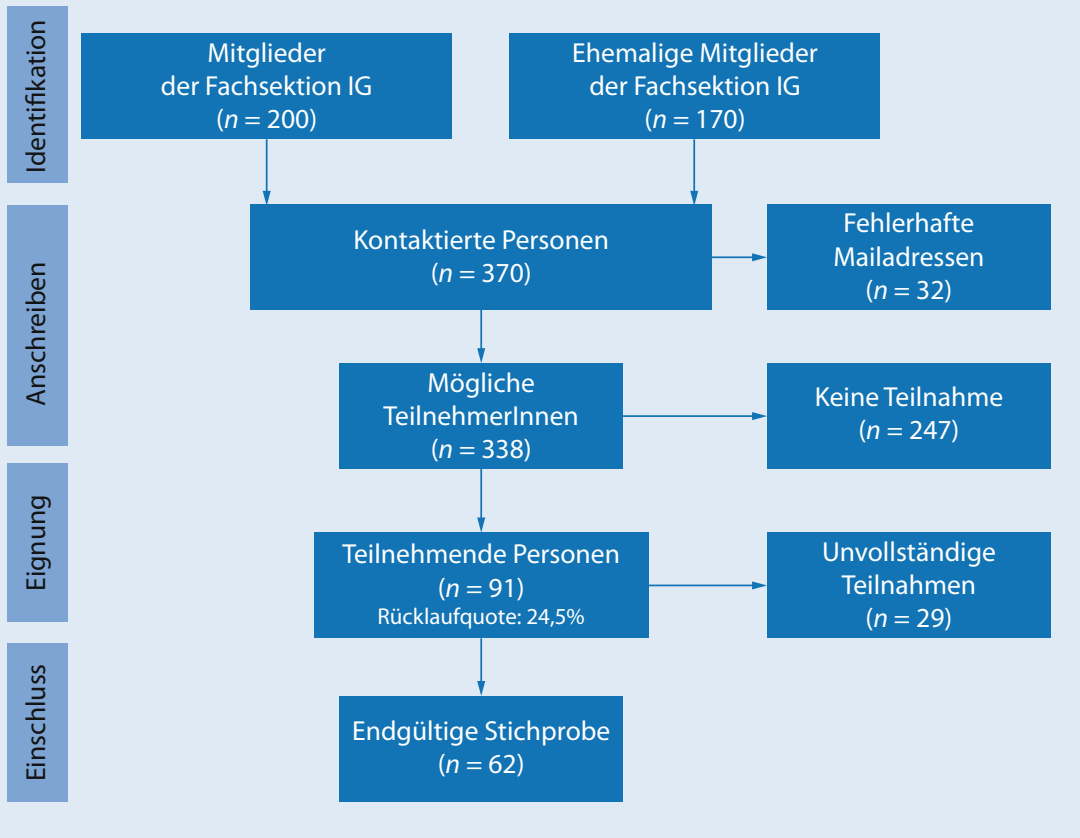

Abb. 1 A Flowchart zur Generierung der Stichprobe. IG integrative Gestalttherapie

findungen, den Verlust von Sympathie oder Achtung für Klienten oder Patienten beim professionellen Helfer" (Burisch 2010, S. 17).

Während Burn-out - als potenzieller Wirkfaktor im „stressful involvement" (Orlinsky und Rønnestad 2005) - den Therapieprozess negativ beeinflussen kann, beschreibt das Kohärenzgefühl als zentrales Element des Salutogenesekonzepts - als potenzieller Wirkfaktor im Sinne des ",healing involvement" (Orlinsky und Rønnestad 2005) - mit positivem Einfluss auf den Therapieprozess, inwieweit eine Person das eigene Leben und Handeln innerlich als stimmig erlebt. Personen mit hohem Kohärenzgefühl bewerten an sie gestellte Anforderungen subjektiv als vorhersehbar, handhabbar und sinnhaft (Reddemann 2014). Folglich geht ein höheres Kohärenzgefühl mit höherem Wohlbefinden bzw. weniger Burn-out-Symptomatik einher (Linley und Joseph 2007). Auch eine längere berufliche Tätigkeit und mehr Arbeitszufriedenheit scheinen mit besserem Wohlbefinden (Reis et al. 2014) bzw. einer geringeren Burn-out-Symptomatik (Lee et al. 2011) verbunden $\mathrm{zu}$ sein. Weitere mögliche Ressourcen von PsychotherapeutInnen stellen die Tätigkeit in Forschung und Lehre, poli- tisches Engagement (Pross 2009) sowie Selbsterfahrung (Reimer 1997) und Supervision bzw. Intervision (Trost 1999) dar. Diese Elemente sind darüber hinaus v. a. für eine kontinuierliche Qualitätssicherung in der Psychotherapie wichtig (Reimer 1997; Strauß und Freyberger 2010; Kanfer et al. 2012).

Die Fachsektion Integrative Gestalttherapie (IG) ist seit Inkrafttreten des österreichischen Psychotherapiegesetzes 1991 ein Teil des Österreichischen Arbeitskreises für Gruppentherapie und Gruppendynamik (ÖAGG). Die IG wird den humanistisch-existenziellen Fachrichtungen der Psychotherapie zugeordnet. Das Fachspezifikum IG ist nicht nur den österreichischen gesetzlichen Vorgaben, sondern einem internationalen Rahmen zur Qualitätssicherung verpflichtet. Laut österreichischem Psychotherapiegesetz $(\$ 2)$ muss vor Eintritt in das Fachspezifikum, das auf eine Psychotherapierichtung fokussiert, ein psychotherapeutisches Propädeutikum absolviert werden, in dem eine übergreifende theoretische und praktische Grundausbildung erfolgt.

Ziel der vorliegenden, weitgehend explorativen Studie war es, bei AbsolventInnen des ÖAGG Fachspezifikum IG Informationen zur Beurteilung der fachspezifischen Ausbildung, zur beruflichen Tätigkeit sowie zu Kohärenzsinn und Burn-out zu erfassen. Damit sollte nicht nur eine Grundlage für die Evaluation und Qualitätssicherung des Fachspezifikums im ÖAGG geschaffen, sondern auch zu einer differenzierteren Sichtweise der Ausbildungslandschaft und der Arbeitsbedingungen von PsychotherapeutInnen beigetragen werden. Der vorliegenden retrospektiven Einschätzung von Input-, Prozess- und Output-Variablen kommt dabei eine große Bedeutung $\mathrm{zu}$, da so die nachhaltige Relevanz von Ausbildungsbestandteilen erfasst werden kann und AbsolventInnen als LehrtherapeutInnen und/oder im Rahmen der Berufspolitik Einfluss auf die zukünftige Gestaltung der Ausbildung nehmen können. Aufbauend auf bisherigen Studien (Linley und Joseph 2007; Lups et al. 2012; Hill und Knox 2013) wurde des Weiteren die Hypothese überprüft, dass ein höherer Kohärenzsinn und eine längere Tätigkeit als PsychotherapeutIn mit einer geringeren Burn-out-Symptomatik in Verbindung stehen.

\section{Material und Methoden}

\section{Studiendesign}

Von Oktober 2015 bis April 2016 nahmen 91 AbsolventInnen des Fachspezifikums IG an der vorliegenden Studie teil. Dies entspricht $24,5 \%$ der per E-Mail kontaktierten Personen (• Abb. 1). Kontaktiert wurden alle aktuellen und ehemaligen Mitglieder der Fachsektion IG. Die Fragebogen wurden mithilfe des LimeSurvey ${ }^{\circledR}$ (LimeSurvey GmbH, Hamburg, Deutschland) vorgegeben; die Beantwortung dauerte etwa $30 \mathrm{~min}$.

\section{Messinstrumente}

$\mathrm{Zu}$ den verschiedenen Aspekten der Ausbildung und Berufsausübung wurde, unter Berücksichtigung bisheriger Studien zu den relevanten Themenbereichen u. a. (Willutzki et al. 1997; Ôrlinsky et al. 1999; Hagleitner und Lang 2005; Reis et al. 2014), in Zusammenarbeit mit der Fachsektion IG des ÖAGG ein allgemeiner soziodemografischer Fragebogen er- 
Psychotherapeut 2018·63:145-152 https://doi.org/10.1007/s00278-017-0245-1

(c) Der/die Autor(en) 2017. Dieser Artikel ist eine Open-Access-Publikation.

\section{Hiebler-Ragger · C. Gollner ·P. Klampfl · L. Nausner · H.-F. Unterrainer \\ Integrative Gestalttherapie in Österreich. Ausbildungs- und Berufsbedingungen, Kohärenzgefühl und Burn-out}

\section{Zusammenfassung}

Hintergrund. Es gibt nur wenige Studien, die sich sowohl mit den verschiedenen Aspekten der Psychotherapieausbildung in Österreich als auch mit dem psychischen Wohlbefinden und der beruflichen Entwicklung von PsychotherapeutInnen beschäftigen.

Ziel der Arbeit. In der vorliegenden, explorativen Arbeit wurden daher AbsolventInnen des Fachspezifikums Integrative Gestalttherapie (IG) des Österreichischen Arbeitskreises für Gruppentherapie und Gruppendynamik (ÖAGG) zu fachspezifischer Ausbildung, beruflicher Situation, Kohärenz- bzw. Burnout-Erleben befragt.

Material und Methoden. Von Oktober 2015 bis April 2016 beantworteten 62 PsychotherapeutInnen einen in Kooperation mit der Fachsektion IG des ÖAGG erstellten Fragebogen, das Maslach Burn-out Inventory (MBI) und die Items zur Sense of Coherence Scale (SOC-29). Der Beginn des Fachspezifikums lag bei den Teilnehmerlnnen durchschnittlich 21 Jahre ( $S D \pm 9,01$ Jahre) zurück, der Abschluss desselben durchschnittlich 13 Jahre ( $S D \pm 9,17$ Jahre).

Ergebnisse. Selbsterfahrung, Lehrtherapie und Lehrsupervision in der Ausbildung wurden als besonders wichtig für die spätere Berufstätigkeit und die persönliche Entwicklung eingeschätzt. Des Weiteren zeigten die Probandlnnen einen überdurchschnittlich hohen Kohärenzsinn $(p<0,01)$ und nur geringe Burn-out-Symptomatik ( $p<0,01)$, wobei ein höherer Kohärenzsinn mit einer geringeren Burn-out-Symptomatik $(p<0,05)$ einherging. Ein Großteil der befragten Personen $(n=40)$ bezeichnete ihre Identität als IG-Therapeutln als hoch ausgeprägt. Ihr berufspolitisches Engagement beschrieben jedoch viele $(n=35)$ als gering. Schlussfolgerung. Auch nach jahrelanger Tätigkeit heben PsychotherapeutInnen die Wichtigkeit der praktischen Erfahrung von Psychotherapie während der Ausbildung hervor. Gleichzeitig scheint eine längere berufliche Tätigkeit mit einer höheren psychischen Belastbarkeit einherzugehen. Zukünftige Studien sollten daher u. a. die Wirkmechanismen von Supervision in Bezug auf die Entwicklung und die Arbeitszufriedenheit von PsychotherapeutInnen genauer untersuchen.

\section{Schlüsselwörter}

Berufszufriedenheit · Belastbarkeit .

Kohärenzsinn · Ressourcen · Supervision

\section{Integrative Gestalt therapy in Austria. Training and professional conditions, sense of coherence and burnout}

Abstract

Background. Only a few studies have been conducted focusing on the different aspects of psychotherapy training in Austria as well as on psychological well-being and the professional development of psychotherapists.

Objective. In this explorative study graduates of the specialist faculty of integrative Gestalt (IG) therapy of the Austrian Working Group for Group Therapy and Group Dynamics (ÖAGG) were therefore questioned regarding their psychotherapy training, their current work situation, as well as their sense of coherence and burnout symptoms.

Material and methods. Between October 2015 and April 2016 a total of 62 psychotherapists completed a sociodemographic questionnaire developed in cooperation with the specialist section of IG therapy of the ÖAGG, together with the Maslach Burnout Inventory (MBI) and the Sense of Coherence Scale (SOC-29). Participants had begun their psychotherapy training on average 21 years ( $S D \pm 9.01)$ ago and completed it on average 13 years $(S D \pm 9.17)$ ago.

Results. Self-experience, training therapy and training supervision were rated as especially important for personal and professional development. Furthermore, the IG therapists also showed a higher than average sense of coherence $(p<0.01)$ that was in turn negatively related to burnout symptoms $(p<0.05)$. Most participants $(n=40)$ reported having a strong identity as an IG therapist; however, they also mostly $(n=35)$ reported a low involvement in professional policy. Conclusion. After years of job experience, psychotherapists still underline the high value of practical experiences during psychotherapy training. In addition, more years of experience as a psychotherapist seem to be related to more resilience. Therefore, future studies should for example aim to further investigate the effects of supervision on the development and job satisfaction of psychotherapists.

Keywords

Job satisfaction - Resilience $\cdot$ Sense of coherence $\cdot$ Resources $\cdot$ Supervison stellt (nähere Informationen dazu über die Erstautorin).

Zur Erfassung von Burn-out wurde das Maslach Burn-out Inventory (MBI; Enzmann und Kleiber 1989) eingesetzt, das emotionale Erschöpfung (EE, 9 Items), Depersonalisation (DP, 5 Items) und persönliche Leistungsfähigkeit (PL, 8 Items) über eine 7-stufige LikertSkala (0: nie bis 6: täglich) erhebt. Als Kerndimensionen des Burn-outs gelten emotionale EE und DP (Taris et al. 2005). Emotionale Erschöpfung definiert sich über Überforderung und die Unfähigkeit zur Entspannung, wodurch sich Müdigkeit, Schlaflosigkeit und diffuse somatische Beschwerden ergeben. Depersonalisation beschreibt die zunehmende Abstumpfung gegenüber dem (Arbeits-)Umfeld, die mit einer zynischen, negativen Einstellung im
Umgang mit Mitmenschen einhergeht (Kapfhammer 2012).

Zur Erfassung des Kohärenzsinns wurde die Sense of Coherence Scale in der Version mit 29 Items (SOC-29; Singer und Brähler 2007) vorgegeben, die über die Aspekte Verstehbarkeit (11 Items), Handhabbarkeit (10 Items) und Sinnhaftigkeit (8 Items) das Gesamtausmaß des Kohärenzsinns erfasst. Da sich die theoretisch begründeten Subskalen faktoren- 
Tab. 1 Deskriptive Ergebnisse zur Bewertung des Fachspezifikums und der beruflichen Tätigkeit Anzahl, $\boldsymbol{n}$ (Anteil, \%)

\begin{tabular}{|c|c|c|c|c|}
\hline & \multicolumn{4}{|c|}{ Anzahl, n (Anteil, \%) } \\
\hline & Keine & Geringe & Mittlere & Hohe \\
\hline \multicolumn{5}{|l|}{ Relevanz verschiedener Aspekte ... } \\
\hline \multicolumn{5}{|l|}{ für die eigene Wahl des Fachspezifikums } \\
\hline Kosten & $23(37,1)$ & $20(32,3)$ & $17(27,4)$ & $2(3,2)$ \\
\hline Zeitlicher Aufwand & $28(45,2)$ & $18(29)$ & $11(17,7)$ & $5(8,1)$ \\
\hline \multicolumn{5}{|l|}{ Zufriedenheit mit ... } \\
\hline Qualität des Fachspezifikums & $0(0)$ & $8(12,9)$ & $19(30,6)$ & $35(65,5)$ \\
\hline $\begin{array}{l}\text { eigenem Einkommen als Psychothera- } \\
\text { peutln }\end{array}$ & $0(0)$ & $5(8,1)$ & $35(65,5)$ & $19(30,6)$ \\
\hline beruflichen Perspektiven & $0(0)$ & $3(4,8)$ & $20(32,3)$ & $36(58,1)$ \\
\hline $\begin{array}{l}\text { Zusammenarbeit mit anderen Berufs- } \\
\text { gruppen }\end{array}$ & $1(1,6)$ & $8(12,9)$ & $22(35,5)$ & $31(50)$ \\
\hline \multicolumn{5}{|l|}{ Ausprägung ... } \\
\hline der Identität als IG-TherapeutIn & $0(0)$ & $6(9,6)$ & $16(25,8)$ & $40(64,5)$ \\
\hline des berufspolitischen Engagements & $17(27,4)$ & $18(29)$ & $16(25,8)$ & $11(17,7)$ \\
\hline
\end{tabular}

Tab. 2 Unterschiede in der Bewertung der Aspekte des Fachspezifikums

\begin{tabular}{|l|l|l|l|l}
\hline Relevanz verschiedener Aspekte ... & M & SD & $\boldsymbol{X}^{2}(\mathbf{4})$ & Rang \\
\hline der IG für die eigene Wahl dieser Richtung & - & - & $166,61^{* *}$ & - \\
\hline Menschenbild & 4,37 & $\pm 1,00$ & - & 1 \\
\hline Methodik & 4,23 & $\pm 1,06$ & - & 1 \\
\hline Persönlichkeitstheorie & 3,05 & $\pm 1,37$ & - & 2 \\
\hline Psychopathologie & 2,18 & $\pm 1,36$ & - & 3 \\
\hline Entwicklungspsychologie & 1,68 & $\pm 1,24$ & - & 4 \\
\hline grundsätzlich für das Fachspezifikum IG & - & - & $58,49^{* *}$ & - \\
\hline Selbsterfahrung & 4,65 & $\pm 0,58$ & - & 1 \\
\hline Lehrtherapie & 4,63 & $\pm 0,55$ & - & 1 \\
\hline Lehrsupervision & 4,39 & $\pm 0,69$ & - & 2 \\
\hline Vermittlung von Methodik & 4,26 & $\pm 0,81$ & - & 2 \\
\hline Theorievermittlung & 3,92 & $\pm 0,89$ & - & 3 \\
\hline für die spätere eigene berufliche Tätigkeit & - & - & $95,33^{* *}$ & - \\
\hline Lehrsupervision & 4,21 & $\pm 0,87$ & - & 1 \\
\hline Lehrtherapie & 4,19 & $\pm 0,81$ & - & 1 \\
\hline Selbsterfahrung & 4,18 & $\pm 0,98$ & - & 1 \\
\hline Vermittlung von Methodik & 3,74 & $\pm 0,85$ & - & 2 \\
\hline Theorievermittlung & 2,81 & $\pm 1,02$ & - & 3 \\
\hline für die eigene persönliche Entwicklung & - & - & $124,10^{* *}$ & - \\
\hline Lehrtherapie & 4,35 & $\pm 0,85$ & - & 1 \\
\hline Selbsterfahrung & 4,27 & $\pm 0,98$ & - & 1 \\
\hline Lehrsupervision & 3,95 & $\pm 0,98$ & - & 2 \\
\hline Vermittlung von Methodik & 3,11 & $\pm 1,09$ & - & 3 \\
\hline Theorievermittlung & 2,65 & $\pm 1,01$ & - & 4 \\
\hline Relevanz: 0: keine bis 5: außerordentlich. Friedman-Tests mit Wilcoxon-Test als Post-hoc-Verfahren, & \\
\hline Rang: Ergebnis des Post-hoc-Verfahrens & & & & \\
\hline IG integrative Gestalttherapie, M Mittelwert, SD Standardabweichung & & \\
\hline * $p$ 0,01 & & & & \\
\hline
\end{tabular}

analytisch nicht reproduzieren lassen, wird die Verwendung des Gesamtsummenwerts empfohlen (Schumacher et al. 2000). Die Items werden über eine 7-stufige Likert-Skala bewertet; die Endpunkte sind jeweils über qualitative Aussagen definiert (z. B.: 1: stimme gar nicht zu bis 7: stimme voll zu; Singer und Brähler 2007).

\section{Stichprobe}

Von 91 TeilnehmerInnen schlossen 62 (42 Frauen) die Befragung ab (• Abb. 1). Diese Daten wurden für die Auswertungen herangezogen. Zum Zeitpunkt der Datenerhebung waren die TeilnehmerInnen zwischen 31 und 67 Jahre alt $(M=$ 52,11 Jahre; $S D \pm 8,70$ Jahre). Der Beginn des Fachspezifikums lag im Durchschnitt 21 Jahre ( $S D \pm 9,01$ Jahre; Range: 5 bis 41 Jahre) zurück, die Eintragung in die Liste der PsychotherapeutInnen des Bundesministeriums für Gesundheit im Durchschnitt 13 Jahre ( $S D \pm 9,17$ Jahre; Range: 0 bis 30 Jahre). Es hatten 46 TeilnehmerInnen (47,2 \%) vor Eintritt in das Fachspezifikum bereits einen akademischen Abschluss.

\section{Statistische Auswertung}

Der Fragebogen zu Ausbildung und Berufsausübung wurde deskriptiv und über Friedman- bzw. Cochran-Tests mit Wilcoxon bzw. McNemar-Tests als Post-hocVerfahren ausgewertet. Zu Burn-out und Kohärenzsinn wurden $t$-Tests und Korrelationen nach Pearson durchgeführt. Das Signifikanzniveau wurde mit $1 \%$ gewählt.

\section{Ergebnisse}

Die deskriptiven Ergebnisse zur Bewertung des Fachspezifikums und der beruflichen Tätigkeit sind in • Tab. 1 aufgeführt.

\section{Beurteilung des Fachspezifikums}

Die anschließenden Analysen (• Tab. 2) zeigten, dass besonders das Menschenbild und die Methodik der IG für die Wahl dieses Fachspezifikums ausschlaggebend waren $\left(\chi^{2}(4)=166,61, p<0,01\right)$. Hinsicht- 
Hier steht eine Anzeige.

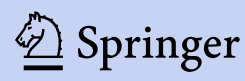


Tab. 3 Unterschiede im Kontakt mit verschiedenen psychotherapeutischen Fachrichtungen

\begin{tabular}{|c|c|c|c|}
\hline Parameter & $\begin{array}{l}\text { Anzahl, } n \\
\text { (Anteil, \%) }\end{array}$ & $x^{2}(5)$ & Rang \\
\hline \multicolumn{4}{|l|}{ (1) Besuch von... } \\
\hline Fortbildungen & $60(96,8)$ & - & - \\
\hline Supervision & $50(80,6)$ & - & - \\
\hline Intervision & $58(93,5)$ & - & - \\
\hline \multicolumn{4}{|c|}{ (2) An welchen psychotherapeutischen Fachrichtungen orientierten sich ... } \\
\hline die besuchten Fortbildungen inhaltlich? & - & $91,96^{* *}$ & - \\
\hline IG & $53(88,3)$ & - & 1 \\
\hline Andere humanistische-existenzielle & $39(65)$ & - & 2 \\
\hline Psychoanalytische & $15(25)$ & - & 3 \\
\hline Tiefenpsychologische & $23(38,3)$ & - & 3 \\
\hline Systemische & $27(45)$ & - & 3 \\
\hline Verhaltenstherapeutische & $7(11,7)$ & - & 4 \\
\hline die konsultierten SupervisorInnen? & - & $58,55^{* *}$ & - \\
\hline IG & $36(72)$ & - & 1 \\
\hline Andere humanistische-existenzielle & $17(34)$ & - & 2 \\
\hline Psychoanalytische & $9(16,1)$ & - & 2 \\
\hline Tiefenpsychologische & $13(23,2)$ & - & 2 \\
\hline Systemische & $11(22)$ & - & 2 \\
\hline Verhaltenstherapeutische & $2(4)$ & - & 3 \\
\hline andere PsychotherapeutInnen in der Intervision? & - & $103,28^{* *}$ & - \\
\hline IG & $49(87,5)$ & - & 1 \\
\hline Andere humanistische-existenzielle & $30(53,6)$ & - & 2 \\
\hline Psychoanalytische & $12(24)$ & - & 4 \\
\hline Tiefenpsychologische & $12(24)$ & - & 4 \\
\hline Systemische & $21(37,5)$ & - & 3 \\
\hline Verhaltenstherapeutische & $8(14,3)$ & - & 4 \\
\hline \multicolumn{4}{|c|}{$\begin{array}{l}\text { Cochran-Test mit McNemar-Test als Post-hoc-Verfahren, \%: Anteil der gesamten (1) bzW. relevan- } \\
\text { ten (2) Fälle, Rang: Ergebnis des Post-hoc-Verfahrens } \\
\text { IG integrative Gestalttherapie } \\
{ }^{* * *} p<0,01\end{array}$} \\
\hline
\end{tabular}

lich der Durchführung des Fachspezifikums wurden der Selbsterfahrung und der Lehrtherapie von allen Bestandteilen die größte Relevanz zugesprochen $\left(\chi^{2}{ }_{(4)}=\right.$ $58,49, p<0,01)$. Des Weiteren wurden Selbsterfahrung, Lehrtherapie und Lehrsupervision auch als relevanter für die spätere berufliche Tätigkeit $\left(\chi_{(4)}^{2}=95,33\right.$, $p<0,01)$ und die eigene persönliche Entwicklung $\left(\chi_{(4)}^{2}=124,10, p<0,01\right)$ eingeschätzt als die Vermittlung von Theorie und Methodik.

\section{Aktuelle Berufstätigkeit}

Die meisten TeilnehmerInnen $(63 \% ; n=$ 39) gaben eine selbstständige Tätigkeit als PsychotherapeutIn an, 4 (6\%) eine unselbstständige. Es arbeiteten 16 (26\%) und besuchte nach dem Fachspezifikum Fortbildungen (97\%; $n=60$ ). Dabei zeigte sich in diesen Bereichen eine überwiegende Treue zur eigenen Fachrichtung, während v. a. zu verhaltenstherapeutischen Richtungen kaum Kontakt bestand (Fortbildungen: $\chi^{2}{ }_{(5)}=91,96$; Supervision: $\chi^{2}(5)=58,55$; Intervision: $\chi^{2}(5)=103,28$; alle $p<0,01$; Tab. 3).

\section{Kohärenzsinn und Burn-out}

Der Kohärenzsinn der TeilnehmerInnen $(M=158,10, S D \pm 16,39)$ war im Vergleich zur Normstichprobe (Schumacher et al. 2000) überdurchschnittlich ausgeprägt $\left(t_{61}=5,98, p<0,01\right)$. Hinsichtlich des Burn-outs zeigten die TeilnehmerInnen (EE: $M=11,18, S D \pm 6,44$; DP: $M=$ 2,81, $S D \pm 2,63 ; \mathrm{PL}: M=42,21, S D \pm 4,75)$ im Vergleich zu deutschen PsychotherapeutInnen (Reis et al. 2014) geringere EE $\left(t_{831}=8,11, p<0,01\right)$ und geringere DP $\left(t_{831}=6,11, p<0,01\right)$. Zur PL lagen aus dieser Studie keine Vergleichswerte vor.

Die Korrelationen zwischen Kohärenzsinn, Burn-out und Dauer der Berufstätigkeit zeigten folgende Ergebnisse: TeilnehmerInnen mit einem höheren Kohärenzsinn gaben eine geringere DP $(r=-0,35, p<0,01)$, eine tendenziell geringere $\mathrm{EE}(r=-0,30, p=0,019)$ und sowie eine tendenziell höhere PL $(r=0,32, p=0,011)$ an. Des Weiteren berichteten TeilnehmerInnen, mit ansteigender Dauer der Berufstätigkeit als PsychotherapeutIn, tendenziell über weniger $\mathrm{EE}(r=-0,28, p=0,029)$ und mehr PL $(r=0,27, p=0,033)$. Die Dauer der Berufstätigkeit wies jedoch keinen Zusammenhang mit dem Ausmaß an DP ( $r=-0,24, p=0,058)$ oder Kohärenzsinn $(r=0,15, p=0,26)$ auf.

\section{Diskussion} Kooperation mit anderen Berufsgruppen im psychosozialen Bereich (15\%; $n=9)$ an. Obwohl die meisten TeilnehmerInnen $(65 \% ; n=40)$ eine stark ausgeprägte Identität als IG-TherapeutIn nannten, führten viele ein geringes $(29 \% ; n=18)$ oder gar kein $(27 \% ; n=17)$ berufspolitisches Engagement an (- Tab. 1).

Ein Großteil der TeilnehmerInnen nahm Supervision ( $81 \% ; n=50)$ bzw. Intervision (94\%; $n=58$ ) in Anspruch
In der vorliegenden Studie wurden PsychotherapeutInnen der Fachrichtung IG zu verschiedenen Aspekten der fachspezifischen Ausbildung, ihrer beruflichen Situation sowie zu Kohärenzsinn und Burn-out befragt. Entsprechend der Aufteilung der Psychotherapieausbildung in Input, Prozess und Output (Strauß und Kohl 2009b) kann als Input festgehalten werden, dass die AbsolventInnen des 
Fachspezifikums IG sich v. a. aufgrund des Menschenbilds und der Methodik für diese Richtung entschieden haben. In Bezug auf den Prozess wiederum gehen v. a. Selbsterfahrung, Lehrtherapie und Lehrsupervision als besonders relevante Ausbildungsbestandteile hervor, die im Sinne des Outputs langfristig als wichtig für die eigene psychotherapeutische Tätigkeit und die eigene persönliche Entwicklung gesehen werden. Zudem ist im Bereich des Outputs eine starke Weiterbeschäftigung mit der eigenen Fachrichtung feststellbar, während $\mathrm{zu}$ anderen Fachrichtungen ein geringerer Kontakt besteht. Dies könnte sich u. a. auf die Bereitschaft auswirken, neue Therapieansätze $\mathrm{zu}$ erlernen (Herschell et al. 2010).

Reimer et al. (2005) sehen positive Berufsperspektiven als wesentliche psychohygienische Einflussvariablen und schließen daraus auf die Notwendigkeit, die eigene Zukunft über aktives Mitwirken in Berufsverbänden zu gestalten. In der vorliegenden Studie äußerten sich die Befragten durchwegs zufrieden mit ihrem Einkommen und ihren beruflichen Perspektiven. Zudem berichteten die meisten über eine stark ausgeprägte Identität als IG-TherapeutIn. Dass das berufspolitische Engagement im Gegensatz dazu als gering eingeschätzt wird, könnte mit der aktuellen Zufriedenheit der TeilnehmerInnen und den folglich wahrscheinlich geringen Wünschen nach Veränderung im Zusammenhang stehen.

Der in anderen Studien (Binder et al. 2006; Brockhouse et al. 2011) berichtete, überdurchschnittlich ausgeprägte Kohärenzsinn bei PsychotherapeutInnen konnte bestätigt werden. Des Weiteren scheint sich mit längerer Berufstätigkeit eine geringere Burn-out-Symptomatik zu zeigen. Dies könnte darauf hinweisen, dass zunehmende Erfahrung das „healing involvement" (Orlinsky und Rønnestad 2005; u.a. das Vertrauen in die eigenen Fähigkeiten sowie konstruktives Coping) fördert, während die Wahrscheinlichkeit von „stressful involvement" (Orlinsky und Rønnestad 2005; u. a. Gefühle von Angst und Langeweile sowie Coping durch Vermeidung) sinkt. Der Einfluss weiterer Variablen auf das psychische Befinden von Psy-
chotherapeutInnen (z. B. Forschung und Lehre [Pross 2009], Supervision oder Intervision [Trost 1999]) sollte in weiteren Studien noch genauer untersucht werden.

\section{Zusammenfassende Betrachtung und Ausblick}

Studien, wie die vorliegende, ermöglichen eine Gegenüberstellung verschiedener Psychotherapierichtungen sowie eine differenziertere Sichtweise der Ausbildungslandschaft und der Arbeitsbedingungen von PsychotherapeutInnen. Dies hat große Bedeutung im Hinblick auf den Zeit- und Kostenaufwand der psychotherapeutischen Ausbildung sowie im Hinblick auf die Qualitätssicherung und Positionierung der Psychotherapie als wichtigen Teil des Gesundheitswesens.

International zeigt sich eine hohe Variabilität in den zur Psychotherapieausbildung zugelassenen Berufsgruppen, der Struktur und Organisation der Ausbildung sowie den anerkannten Fachrichtungen (Strauß und Kohl 2009b). Dies muss bei der Interpretation und der Gegenüberstellung der Ergebnisse mit anderen Studien jedenfalls berücksichtigt werden. Des Weiteren wäre für eine umfassende Beurteilung der Qualität und der Auswirkungen des Fachspezifikums IG des ÖAG v. a. auch die laufende Evaluation über die gesamte Ausbildungsdauer bis in die Berufstätigkeit sinnvoll. Über die Veröffentlichung solcher Evaluationen durch verschiedene Ausbildungsinstitute könnte in der Folge die Qualität der Ausbildung weiter verbessert werden. Als weitere Einschränkung ist anzuführen, dass systematische Unterschiede zwischen TeilnehmerInnen und NichtteilnehmerInnen der vorliegenden Studie nicht untersucht werden konnten. Auch unvollständige Teilnahmen, die durchwegs durch Abbrüche zu Beginn der Befragung entstanden, konnten nicht sinnvoll in den Auswertungen berücksichtigt werden. Es besteht daher die Möglichkeit, dass die vorliegenden Ergebnisse nicht für alle AbsolventInnen dieses Fachspezifikums Gültigkeit haben. Des Weiteren konnte bei der vorliegenden Stichprobengröße nicht adäquat für die große Heterogenität (z. B. in den Jahren der Berufstätigkeit) kontrolliert werden. Auch kann das absolute Ausmaß an Burn-out in der vorliegenden Stichprobe aufgrund fehlender deutschsprachiger Cut-off-Werte nicht beurteilt werden.

Basierend auf den erhobenen Daten kann festgehalten werden, dass AbsolventInnen des Fachspezifikums IG des ÖAGG die Bedeutung der praktischen Erfahrung von Psychotherapie in der Ausbildung (Supervision, Selbsterfahrung und Lehrtherapie) für die spätere berufliche Tätigkeit besonders hervorheben. Auch Orlinsky und Rønnestad (2005) sehen diese Bereiche als wichtig für die Kompetenzförderung an, während Hill und Knox (2013, S. 778) ausführen, dass das Ziel der Ausbildung nicht im Erwerb von Fähigkeiten besteht, sondern darin zu lernen, wann und warum diese Fähigkeiten abgestimmt auf einzelne KlientInnen eingesetzt werden sollen. Dies wiederum könnte auch die eher uneinheitliche Einschätzung der Relevanz der Theorievermittlung für die berufliche Tätigkeit und die persönliche Entwicklung erklären.

Auch wenn bei den TeilnehmerInnen der Beginn des Fachspezifikums im Durchschnitt 21 Jahre und der Abschluss im Durchschnitt 13 Jahre zurücklagen, kommt dieser retrospektiven Einschätzung von Input-, Prozess- und OutputVariablen doch große Bedeutung zu, da so die nachhaltige Relevanz von Ausbildungsbestandteilen erfasst werden kann und ehemalige AbsolventInnen als LehrtherapeutInnen und/oder im Rahmen der Berufspolitik Einfluss auf die zukünftige Gestaltung der Ausbildung nehmen können. Um einen besseren Einblick in die Mechanismen und Auswirkungen der Psychotherapieausbildung zu bekommen, bedarf es jedenfalls einer längsschnittlichen, auf diesen ersten Erkenntnissen aufbauenden Evaluation des Ausbildungsverlaufes. Insbesondere wäre interessant, ob sich die Einschätzung der Relevanz verschiedener Aspekte der Ausbildung für berufliche Tätigkeit und persönliche Entwicklung im Ausbildungsverlauf bzw. mit zunehmender beruflicher Tätigkeit verändert. 


\section{Fazit für die Praxis}

\section{- Selbsterfahrung, Lehrtherapie und Lehrsupervision haben anhaltende Relevanz für persönliche Entwicklung und berufliche Praxis. \\ - Kohärenzsinn und Berufserfahrung gehen zumindest tendenziell mit ei- ner geringeren Burn-out-Problematik einher. \\ - Neben einem hohen Identitäts- bewusstsein besteht ein geringes berufspolitisches Engagement in der untersuchten Gruppe von Gestalt- PsychotherapeutInnen. \\ - Als sinnvoller nächster Schritt zur Qualitätssicherung in der Psycho- therapieausbildung wird auf die Möglichkeit längsschnittlicher Eva- luationen verwiesen.}

\section{Korrespondenzadresse}

PD Dr.habil. Dr.habil. H.-F. Unterrainer

Zentrum für Integrative Suchtforschung Verein Grüner Kreis

Rudolfsplatz 9, 1090 Wien, Österreich

human.unterrainer@gruenerkreis.at

Funding. Open access funding provided by Medical University of Graz.

\section{Einhaltung ethischer Richtlinien}

Interessenkonflikt. M. Hiebler-Ragger, C. Gollner, P. Klampfl, L. Nausner und H.-F. Unterrainer geben an, dass kein Interessenkonflikt besteht.

Alle im vorliegenden Manuskript beschriebenen Befragungen wurden mit Zustimmung der Ethikkommission der Karl-Franzens-Universität Graz, im Einklang mit nationalem Recht sowie gemäß der Deklaration von Helsinki von 1975 (in der aktuellen, überarbeiteten Fassung) durchgeführt. Von allen Beteiligten liegt eine Einverständniserklärung vor.

Open Access. Dieser Artikel wird unter der Creative Commons Namensnennung 4.0 International Lizenz (http://creativecommons.org/licenses/by/4.0/deed. de) veröffentlicht, welche die Nutzung, Vervielfältigung, Bearbeitung, Verbreitung und Wiedergabe in jeglichem Medium und Format erlaubt, sofern Sie den/die ursprünglichen Autor(en) und die Quelle ordnungsgemäßnennen, einen Link zur Creative Commons Lizenz beifügen und angeben, ob Änderungen vorgenommen wurden.

\section{Literatur}

Binder HP, Mesenholl-Strehler E, Pass P, Endler PC (2006) Sense of coherence (SOC) among psychotherapists in Austria, differentiated according to number of individually completed training therapy sessions. TSW Holist Health Med 1:232-235

Brockhouse R, Msetfi RM, Cohen K, Joseph S (2011) Vicarious exposure to trauma and growth in therapists: the moderating effects of sense of coherence, organizational support, and empathy. JTrauma Stress 24:735-742

Burisch M (2010) Das Burnout-Syndrom - Theorie de inneren Erschöpfung, 4. Aufl. Springer, Berlin, Heidelberg

Enzmann D, Kleiber D (1989) Helfer-Leiden: Stress und Burnout in psychosozialen Berufen. Asanger, Heidelberg

Hagleitner J, Lang M (2005) Psychodramatherapie-Ausbildung im fachspezifischen Vergleich innerhalb Österreichs: eine retrospektive Befragung der AbsolventInnen. Psychother Forum 13:26-35

Herschell $A D$, Kolko DJ, Baumann BL, Davis AC (2010) The role of therapist training in the implementation of psychosocial treatments: a review and critique with recommendations. Clin Psychol Rev 30:448-466

Hill CE, Knox S (2013) Training and supervision in psychotherapy: evidence for effective practice. In: Lambert MJ (Hrsg) Handbook of psychotherapy and behavior change. Wiley, New York, S775-811

Hoffmann N, Hoffmann B (2008) Selbstfürsorge für Therapeuten und Berater. Beltz, Weinheim

Jaeggi E (2001) Und wer therapiert die Therapeuten? 3. Aufl. Klett-Cotta, Stuttgart

Kanfer FH, Reinecker H, Schmelzer D (2012) Selbstmanagement-Therapie, 5. Aufl. Springer, Berlin, Heidelberg

Kapfhammer H (2012) Burnout - Krankheit oder Symptom? Internist 53:1276-1288

Laireiter AR, Botermans J-F (2005) Ausbildungsforschung in der Psychotherapie - Entwicklungen und aktueller Stand. In: Laireiter AR, Willutzki $\mathrm{U}$ (Hrsg) Ausbildung in Verhaltenstherapie. Hogrefe, Göttingen, S53-101

Lee J, Nayoung Lim, Eunjoo Y, Lee SM (2011) Antecedents and consequences of three dimensions of burnout in psychotherapists: a meta-analysis. Prof Psychol Res Pr 42:252-258

Linley PA, Joseph S (2007) Therapy work and therapists' positive and negative well-being. J Soc Clin Psychol 26:385-403

Lups F, KliemS, KrögerC (2012) Sekundäre Traumatisierung. Eine Umfrage unter Psychotherapeuten. Psychotherapeutenjournal 2:94-99

Ôrlinsky D et al (1999) Development of psychotherapists: concepts, questions, and methods of a collaborative international study. Psychother Res 9:127-153

Orlinsky DE, Rønnestad MH (2005) How psychotherapists develop: a study of therapeutic work and professional growth. American Psychological Association, Washington, DC

Pross C (2009) Verletzte Helfer. Umgang mit dem Trauma: Risiken und Möglichkeiten sich zu schützen. Klett-Cotta, Stuttgart

Reddemann L (2014) Psychodynamisch Imaginative Traumatherapie (PITT). In: Maercker A (Hrsg) Posttraumatische Belastungsstörungen. KlettCotta, Stuttgart, S281-296

Reimer C (1997) Gefahren bei der Ausübung des psychotherapeutischen Berufes. Psychotherapeut 42:307-313

Reimer C, Jurkat HB, Vetter A, Raskin K (2005) Lebensqualität von ärztlichen und psycholo- gischen Psychotherapeuten. Psychotherapeut 50:107-114

Reis D, Schröder A, Schlarb A (2014) Wohlbefinden, Burn-out und Ressourcen bei Psychotherapeuten. Psychotherapeut 59:46-51

Ronnestad MH, Skovholt TM (2013) The developing practitioner: growth and stagnation of therapists and counselors, 1. Aufl. Routledge, New York

Schumacher J, Gunzelmann T, Brähler E (2000) Deutsche Normierung der Sense of Coherence Scale von Antonovsky. Diagnostica 46:208-213

Singer S, Brähler E (2007) Die "Sense of Coherence Scale" - Testhandbuch zur deutschen Version. Vandenhoeck\& Ruprecht, Göttingen

StraußB, Freyberger HJ (2010) Supervision als zentraler Bestandteil professioneller Psychotherapie. Psychotherapeut 55:453-454

Strauß B, Kohl S (2009a) Entwicklung der Psychotherapie und der Psychotherapieausbildung in europäischen Ländern: Ergebnisse einer Expertenbefragung. Psychotherapeut 54:457-464

Strauß B, Kohl S (2009b) Themen der Ausbildungsforschung in der Psychotherapie. Psychotherapeut 54:411-426

Taris TW, LeBlancPM, Schaufeli WB, Schreurs PJG (2005) Are there causal relationships between the dimensions of the Maslach Burnout Inventory? A review and two longitudinal tests. Work Stress 19:238-255

Trost A (1999) Psychohygiene - Hilfe für Helfer. In: Schwarzer W, Trost A (Hrsg) Psychiatrie und Psychotherapie für psychosoziale und pädagogische Berufe. Bergmann, Dortmund, S405-423

Willutzki U, Jean-Francois Botermans, SPR Collaborative Research Network (1997) Ausbildung in Psychotherapie in Deutschland und der Schweiz und ihre Bedeutung für die therapeutische Kompetenz. Psychotherapeut 42:282-289 